\title{
Gemcitabine and Docetaxel Combination for Advanced Soft Tissue Sarcoma: A Nationwide Retrospective Study
}

\section{Yunjung Choi, MPH,2 \\ Mi Sun Yun, RN ${ }^{1}$ \\ Sang Hee Lim, MS ${ }^{1}$ \\ Jeeyun Lee, MD, $\mathrm{PhD}^{3}$ \\ Jin-Hee Ahn, MD, $P h D^{4}$ \\ Yu Jung Kim, MD, $P h D^{5}$ \\ Kyong Hwa Park, MD, PhD 6 \\ Young Suk Park, MD, PhD $3{ }^{3,7}$ \\ Ho Yeong Lim, MD, PhD $3{ }^{3,7}$ \\ Hyonggin An, $\mathrm{PhD}^{8}$ \\ Dong-Churl Suh, PhD² \\ Yeul Hong Kim, MD, PhD,}

${ }^{*}$ A list author's affiliations appears at the end of the paper.
Correspondence: Yeul Hong Kim, MD, PhD

Division of Oncology/Hematology,

Department of Internal Medicine,

Korea University College of Medicine,

73 Inchon-ro, Seongbuk-gu, Seoul 02841, Korea

Tel: 82-2-920-5569

Fax: 82-2-926-4534

E-mail: yhk0215@korea.ac.kr

Co-correspondence: Dong-Churl Suh, $\mathrm{PhD}$ College of Pharmacy Chung-Ang University, 84 Heukseok-ro, Dongjak-gu, Seoul 06974, Korea Tel: $82-2-820-5600$

Fax: 82-2-813-0650

E-mail: dongsuh75@gmail.com

Received November 10, 2016

Accepted March 15, 2017

Published Online March 30, 2017

\section{Purpose}

This nationwide retrospective study was conducted to evaluate the efficacy and safety of combined gemcitabine and docetaxel (GD) as an off-label therapy for advanced soft tissue sarcoma, which has limited treatment options owing to its rare occurrence.

\section{Materials and Methods}

A total of 228 patients received GD therapy for advanced soft tissue sarcoma from 2009 to 2014 in Korea. We retrospectively reviewed the clinical medical records and claims data of these patients.

\section{Results}

A total of 218 patients in 20 medical centers were included in the final analysis (median age, 50.0 years). The objective response rate was $15.1 \%$ (34/218, in the leiomyosarcoma subgroup; 26.3\%). The median overall survival and progression-free survival were 10.3 months (95\% confidence interval [Cl], 8.4 to 12.2$)$ and 3.3 months $(95 \% \mathrm{Cl}, 2.8$ to 4.7$)$, respectively. The treatment was discontinued in $7.8 \%$ of patients owing to adverse events; however, there was no adverse event-related death. Neutropenia (35.7\%) and anemia (15.1\%) were the most frequent grade 3/4 toxicities. Univariate analysis for identifying the predictors of the progression-free survival period revealed that patients aged $\leq 50$ years had a hazard ratio of $1.388(95 \% \mathrm{Cl}, 1.027$ to $1.875 ; \mathrm{p}<0.05)$ relative to those aged $>50$ years, and the group with leiomyosarcoma had a hazard ratio of 0.693 (95\% Cl, 0.493 to $0.975 ; p<0.05)$ relative to the group with other histopathological subtypes.

\section{Conclusion}

GD therapy was tolerable and effective for Korean patients with soft tissue sarcoma. In conclusion, for patients with advanced soft tissue sarcoma, especially leiomyosarcoma, GD therapy could be an important therapeutic option.

\author{
Key words \\ Advanced soft tissue sarcoma, Gemcitabine, Docetaxel, \\ Off-label use, Retrospective study
}




\section{Introduction}

Soft tissue sarcoma refers to a type of malignant tumor that originates in the mesodermal tissues. This condition accounts for less than $1 \%$ of all cancers that occur in humans and is classified into 50 or more histopathological subtypes, such as leiomyosarcoma (LMS), liposarcoma, and others [1,2]. Anthracycline-based chemotherapy (e.g., doxorubicin and ifosfamide combination) is the most commonly used firstline drug therapy for soft tissue sarcoma [3-5], and when such therapy fails, high-dosage ifosfamide, pazopanib alone, or gemcitabine and docetaxel combination is administered [1,6-10].

Among the second-line therapies for soft tissue sarcoma, gemcitabine and docetaxel combination is an off-label regimen that can only be used in approved hospitals in Korea. Gemcitabine and docetaxel have been not approved for advanced soft tissue sarcoma by the Korea Ministry of Food and Drug Safety. However, this regimen has been in off-label use since February 2009 with prior approval from the Korean Health Insurance Review \& Assessment Service (HIRA), and as of 2014, it has been used for 228 cases. In Korea, patients with advanced soft tissue sarcoma who failed anthracyclinebased chemotherapy were usually administered a gemcitabine and docetaxel combination between 2009 and 2014.

This Korean nationwide retrospective study was conducted to evaluate the efficacy and safety of combined gemcitabine and docetaxel as an off-label therapy for advanced soft tissue sarcoma.

\section{Materials and Methods}

\section{Patients}

A total of 228 patients received combined gemcitabine and docetaxel for advanced soft tissue sarcoma at 20 medical centers beginning in February 2009, when it was first approved as an off-label regimen, and continuing until 2014. The patients were diagnosed with soft tissue sarcoma and received one or more anticancer therapies that failed; therefore, they subsequently received gemcitabine and docetaxel combination therapy for cancer metastasis or recurrence.

In these patients, the histopathological subtypes included alveolar soft-part sarcoma, desmoplastic small round cell tumor, epithelioid sarcoma, gastrointestinal stromal tumor, neuroectodermal tumor, fibrosarcoma, LMS, liposarcoma, malignant fibrous histiocytoma, malignant hemangiopericytoma, malignant peripheral nerve sheath tumor, and syn- ovial sarcoma. Because clear cell sarcoma, extraskeletal chondrosarcoma, extraskeletal osteosarcoma, and Ewing's sarcoma/primitive neuroectodermal tumors are histopathological types not approved for this off-label regimen by HIRA, patients with these subtypes were excluded from this study.

This study was approved by the Institutional Review Board of the HIRA in Korea (2015-013-001).

\section{Data collection}

Twenty medical centers that were approved for the offlabel use of combined gemcitabine and docetaxel had their patient medical records provided to HIRA for evaluation of off-label use. Data were collected from the medical records of these patients. Information regarding the patients' demographic characteristics, such as age and sex; disease characteristics, such as histopathological classification and site of origin; treatment effects; and adverse events was obtained. In addition, health insurance claims data for the use of gemcitabine and docetaxel combination were reviewed to confirm the patient's age, date of disease progression and date of death.

\section{Chemotherapy treatment}

The patients were administered (1) a single cycle of gemcitabine 1,000 $\mathrm{mg} / \mathrm{m}^{2}$ intravenously (D1, D8) and docetaxel $35 \mathrm{mg} / \mathrm{m}^{2}$ intravenously (D1, D8) every 21 days or (2) gemcitabine $900 \mathrm{mg} / \mathrm{m}^{2}$ intravenously (D1, D8) and docetaxel 75$100 \mathrm{mg} / \mathrm{m}^{2}$ intravenously (D1) every 21 days. When administering this regimen, each medical center performed a drug response evaluation every $2-3$ cycles. The drug administration was discontinued if the disease progressed or a serious adverse event occurred, and initiating the next cycle of chemotherapy was allowed if tumors showed complete response (CR), partial response (PR), or stable disease (SD).

\section{Efficacy}

Efficacy was based on the Response Evaluation Criteria In Solid Tumors (RECIST) ver. 1.1 performed by each medical center. Based on the best tumor response observed after therapy, tumor responses were classified as CR, PR, SD, or progressive disease (PD). The objective tumor response rate was defined as $\mathrm{CR}+\mathrm{PR}$. Cases that did not belong to any of these classifications or when a tumor response could not be evaluated were marked as not evaluable. The progression-free survival (PFS) period was defined as the time from the start of the therapy to the date of disease progression or death. 


\section{Toxicity}

Hematological adverse events were evaluated using National Cancer Institute Common Terminology Criteria for Adverse Events (NCI-CTCAE) ver. 4.0 with reference to hematocrit, white blood cell (WBC), and platelet counts. In addition, nonhematological adverse events were obtained from the patients' medical records.

\section{Statistical analysis}

Statistical analysis of the data was conducted using SAS EG 4.3 (SAS Institute Inc., Cary, NC). The patients' characteristics, response rates, and adverse events were analyzed. Survival was analyzed using the Kaplan-Meier survival estimation method. In addition, the Cox proportional hazards model was applied to analyze the prognosticators of PFS and overall survival, with $\mathrm{p}<0.05$ set as the statistical significance level.

\section{Results}

\section{Patient characteristics}

A total of 218 patients from 20 medical centers were included in the final analysis. From all initially reported cases, 10 were excluded, including nine with unreliable histopathological reports and one case of patient information entry error. The patient population consisted of 111 men (50.9\%) and 107 women $(49.1 \%$ ) with a median age of 50.0 years (range, 18 to 77 years). The percentage of patients with metastasis and recurrence was $71.4 \%$ and $28.1 \%$, respectively. With respect to performance status, the majority of the patients $(81.7 \%)$ had an Eastern Cooperative Oncology Group (ECOG) performance status score of 0 or I, while $12.4 \%$ and $2.3 \%$ of patients had ECOG scores of II and III, respectively. Patients who had undergone surgical treatment accounted for $82.1 \%$, while $50.5 \%$ had undergone radiation therapy, 58.1\% received one type of chemotherapy, and $40.9 \%$ received two or more types of chemotherapy. The abdomen-retroperitoneum-pelvis was the most common site of cancer origin (49.1\%), followed in order by the extremities, chest, internal organs, other sites, and head and neck (Table 1).

\section{Efficacy}

The percentage of patients who had a dose reduction was $45.8 \%$, and the actual dose intensity received was $95 \%$ of the
Table 1. Baseline demographic and clinical characteristics of patients

\begin{tabular}{|c|c|}
\hline Characteristic & No. $(\%)$ \\
\hline \multicolumn{2}{|l|}{ Sex } \\
\hline Male & $111(50.9)$ \\
\hline Female & $107(49.1)$ \\
\hline Age, median (range, yr) & $50(18-77)$ \\
\hline \multicolumn{2}{|l|}{ Histology } \\
\hline Leiomyosarcoma & $57(26.1)$ \\
\hline Malignant fibrous histiocytoma & $36(16.5)$ \\
\hline Liposarcoma & $27(12.4)$ \\
\hline Synovial sarcoma & $19(8.7)$ \\
\hline Malignantperipheral nerve sheath tumor & $15(6.9)$ \\
\hline Fibrosarcoma & $12(5.5)$ \\
\hline Epithelioid sarcoma & $9(4.1)$ \\
\hline Angiosarcoma & $8(3.7)$ \\
\hline Desmoplastic small round cell tumor & $6(2.8)$ \\
\hline Malignant hemangiopericytoma & $5(2.3)$ \\
\hline Alveolar soft part sarcoma & $2(0.9)$ \\
\hline Neuroectodermal tumor & $1(0.5)$ \\
\hline Sarcoma, not otherwise specified & $21(9.6)$ \\
\hline \multicolumn{2}{|l|}{ Initial localization } \\
\hline Abdomen-retroperitoneum-pelvis & $107(49.1)$ \\
\hline Extremity & $50(22.9)$ \\
\hline Chest & $37(17.0)$ \\
\hline Head and neck & $10(4.6)$ \\
\hline Others & $14(6.4)$ \\
\hline \multicolumn{2}{|l|}{ Previous treatment } \\
\hline Surgery & $179(82.1)$ \\
\hline Adjuvant chemotherapy & $56(25.7)$ \\
\hline Radiotherapy & $110(50.5)$ \\
\hline Palliative chemotherapy & $210(96.3)$ \\
\hline \multicolumn{2}{|l|}{ No. of previous chemotherapy lines } \\
\hline 1 & $129(59.2)$ \\
\hline 2 & $52(23.9)$ \\
\hline$\geq 3$ & $37(17.0)$ \\
\hline \multicolumn{2}{|l|}{ ECOG PS } \\
\hline 0 & $34(15.6)$ \\
\hline 1 & $145(66.1)$ \\
\hline 2 & $27(12.4)$ \\
\hline 3 & $5(2.3)$ \\
\hline Unknown & $8(3.7)$ \\
\hline
\end{tabular}

ECOG PS, Eastern Cooperative Oncology Group performance status.

dose intensity approved by the HIRA. The primary cause of the dose reduction was adverse events $(52.6 \%)$, with approximately $19 \%$ occurring because of old age or patient condition. In $3.7 \%$ of the cases, the dose was slightly increased and the number of chemotherapy cycles was three. Among the 218 
Table 2. Responses to gemcitabine and docetaxel combination treatment

\begin{tabular}{lc} 
Response & No. $(\%)$ \\
Complete response & $1(0.5)$ \\
Partial response & $33(15.1)$ \\
Stable disease & $79(36.2)$ \\
Progressive disease & $83(38.1)$ \\
Not evaluable & $22(10.1)$ \\
Objective response rate & $34(15.6)$ \\
\hline
\end{tabular}

patients who were included in the final analysis, 196 had RECIST reports available, while response evaluation could not be performed in 22 patients because of early termination of treatment or other reasons.

For all 218 patients, tumor response evaluations were based on the best response among all evaluations. The results showed one patient with CR $(0.5 \%), 33$ patients with PR $(15.1 \%), 83$ patients with SD $(38.1 \%)$, and 83 patients with PD (38.1\%), indicating that the objective tumor response rate was 15.6\% (Table 2). Among the histopathological subtypes, LMS showed a higher response rate of $26.3 \%$ (15/57), the response rate for malignant fibrous histiocytoma was $11.1 \%(4 / 35)$, that for liposarcoma was $14.8 \%(4 / 27)$, and that for synovial sarcoma was 10.5\% (2/19) (Table 3). With regard to drug administration, the 1-week regimen, in which docetaxel was administered twice at intervals of 1 week (gemcitabine 1,000 mg/ $\mathrm{m}^{2}$, D1, D8; docetaxel $35 \mathrm{mg} / \mathrm{m}^{2}$, D1, D8), resulted in a higher response rate of $19 \%(20 / 104)$, while the response rate was
$11.7 \%$ (13/111) for the 3-week regimen, where docetaxel was administered every 3 weeks (gemcitabine 1,000 mg/ ${ }^{2}$, D1, D8+docetaxel 75-100 mg/m² D1).

The median PFS and overall survival durations analyzed using the Kaplan-Meier survival estimation method were 3.3 months (95\% confidence interval [CI], 2.8 to 4.7 ) and 10.3 months (95\% CI, 8.4 to 12.2), respectively (Figs. 1 and 2) in the median follow-up period of 343 days. There were no significant differences among tissue types, but there was a significant PFS difference between LMS (median PFS, 5.8 months) and all other tissue types (median PFS, 3.0 months) (Fig. 3).

Univariate analysis for identifying the predictors of the PFS period revealed that patients aged $\leq 50$ years had a hazard ratio of $1.388(95 \% \mathrm{CI}, 1.027$ to $1.875 ; \mathrm{p}<0.05)$ relative to those aged $>50$ years, and that those with LMS $(\mathrm{n}=57$; median age, 50.0 ) had a hazard ratio of 0.693 (95\% CI, 0.493 to $0.975 ; \mathrm{p}<$ 0.05 ) relative to the group with other histopathological subtypes ( $\mathrm{n}=160$; median age, 50.0) (Table 4 ).

\section{Toxicity}

Hematological adverse events were evaluated using NCICTCAE ver. 4.0 with reference to hematocrit, WBC, and platelet counts from the clinical evaluations performed by each medical center. The results showed grade 3 (G3) or higher adverse events of neutropenia (78 of 218 patients, $35.7 \%$ ), anemia (15.1\%), thrombocytopenia (11.4\%), and febrile neutropenia $(7.4 \%)$ (Table 5). In addition, G3 or higher nonhematological adverse events reported included nausea/vomiting $(1.4 \%)$, general weakness $(1.4 \%)$, fatigue $(1.4 \%)$, myalgia $(1.0 \%)$, and skin rash (1.4\%). Discontinuation of the drug

Table 3. Objective response rate by histology

\begin{tabular}{lcc} 
Variable & $\begin{array}{c}\text { Objective } \\
\text { response rate (\%) }\end{array}$ & No./Total \\
\hline All histological subtypes & 15.7 & $34 / 217$ \\
Leiomyosarcoma & 26.3 & $15 / 57$ \\
Malignant fibrous histiocytoma & 11.1 & $4 / 35$ \\
\hline Liposarcoma & 14.8 & $4 / 27$ \\
\hline Synovial sarcoma & 10.5 & $2 / 19$ \\
Malignant peripheral nerve sheath tumor & 0 & $0 / 16$ \\
\hline Fibrosarcoma & 16.7 & $2 / 12$ \\
\hline Epithelioid sarcoma & 11.1 & $1 / 9$ \\
Angiosarcoma & 37.5 & $3 / 8$ \\
\hline Desmoplastic small round cell tumor & 16.7 & $1 / 6$ \\
\hline Malignant hemangiopericytoma & 0 & $0 / 5$ \\
\hline Alveolar soft part sarcoma & 0 & $0 / 2$ \\
\hline Neuroectodermal tumor & 0 & $0 / 1$ \\
Sarcoma, not otherwise specified & 9.5 & $2 / 21$ \\
\hline
\end{tabular}


Table 4. Prognostic factors for progression-free survival: univariate analysis with Cox univariate models

\begin{tabular}{lcc} 
& Hazard ratio (95\% CI) & p-value \\
\hline Performance status (0-1 vs. 2-3) & $0.694(0.452-1.066)$ & 0.0954 \\
No. of lines of previous systemic therapy (1 vs. $\geq 2)$ & $0.888(0.655-1.205)$ & 0.4475 \\
Sex (female vs. male) & $1.247(0.925-1.681)$ & 0.1476 \\
Age ( $\leq 50$ yr vs. $>50$ yr) & $1.388(1.027-1.875)$ & 0.0326 \\
Histology subtype & $0.693(0.493-0.975)$ & 0.0352 \\
$\quad$ (leiomyosarcoma vs. other sarcoma) & & \\
\hline
\end{tabular}

CI, confidence interval.

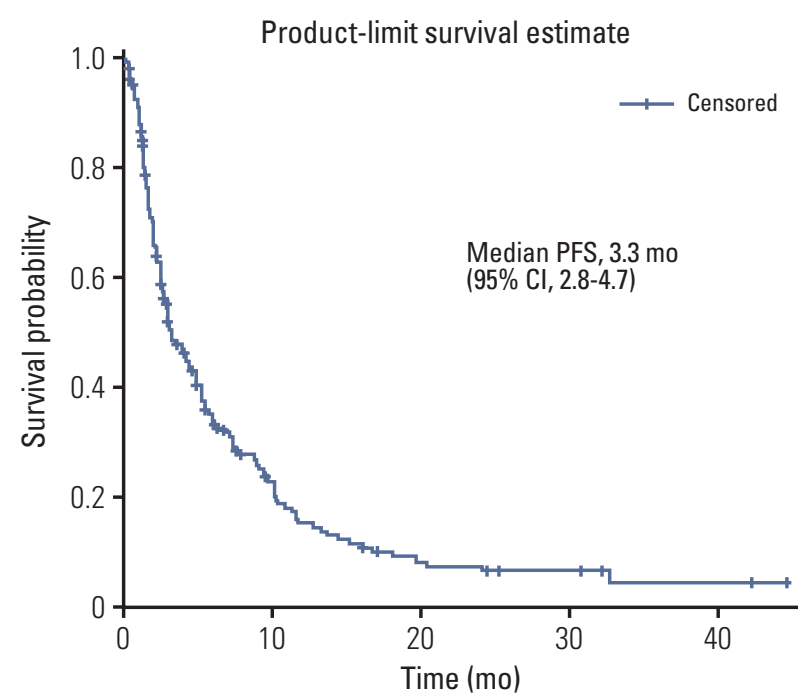

Fig. 1. Kaplan-Meier estimates of progression-free survival (PFS) in patients treated with gemcitabine and docetaxel combination as a second or subsequent line treatment in Korea. CI, confidence interval.

owing to adverse events occurred in $7.8 \%$ of the cases; however, there was no adverse event-related death.

\section{Discussion}

Soft tissue sarcoma, a relatively rare cancer, has 50 or more histopathological subtypes, such as LMS and liposarcoma, and it may appear heterogeneous depending on the histopathological subtype $[1,6,7]$. Only a few effective chemotherapy regimens are currently available for treatment of advanced soft tissue sarcoma, and there have been very few phase III clinical trials of second-line chemotherapy [6-10].

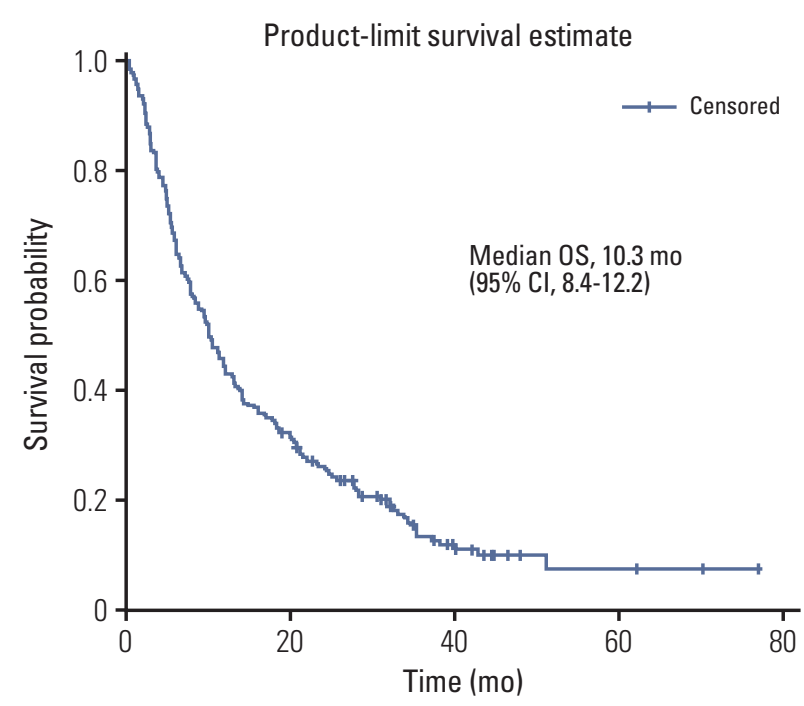

Fig. 2. Kaplan-Meier estimates of overall survival (OS) in patients treated with gemcitabine and docetaxel combination as a second or subsequent line treatment in Korea. CI, confidence interval.

There have been a few clinical trials of gemcitabine and docetaxel combination therapy, which is recommended as a second-line chemotherapy regimen for soft tissue sarcoma; however, the largest retrospective study reported to date is the 2006 French study of 133 patients with soft tissue sarcoma from 10 institutions [11]. In that study, the overall response to gemcitabine and docetaxel combination therapy was $18.4 \%$ and the median overall survival was 12.1 months. In the current study, which examined 218 Korean patients, the objective response rate was $15.6 \%$, while the median overall survival was 10.3 months. It is difficult to compare the results from these two studies since they evaluated patients from different countries in different clinical settings. However, both studies are significant because they were relatively large multicenter studies that reflected real clinical settings and 


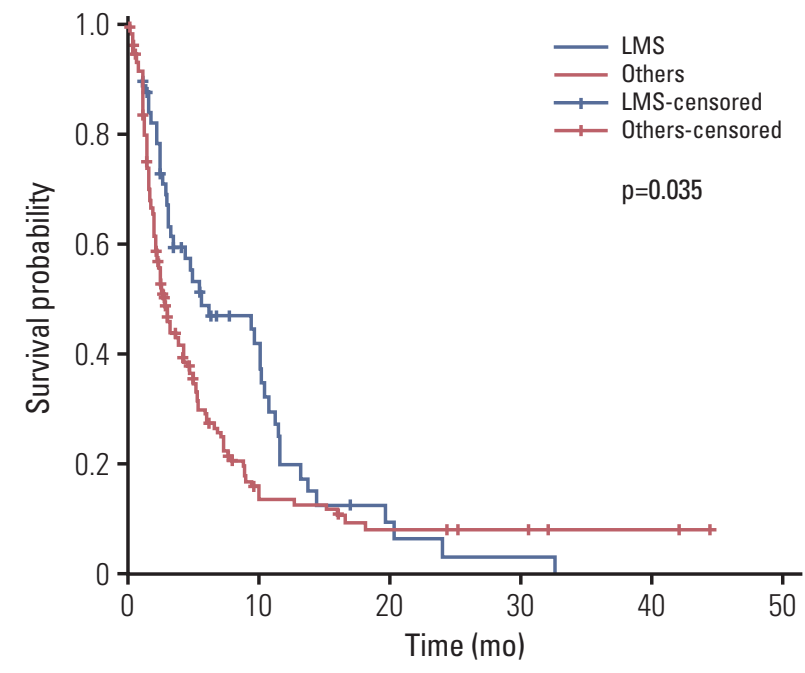

Fig. 3. Kaplan-Meier estimates of progression-free survival in patients treated with gemcitabine and docetaxel combination as a second or subsequent line treatment according to histology in Korea. LMS, leiomyosarcoma. evaluated a rare cancer for which it is difficult to perform phase III clinical trials.

In the French study, uterine LMS, a histopathological subtype that is relatively more responsive, accounted for a significantly higher proportion $(57.1 \%)$ of cases, and the subtypes of uterine LMS showed higher response rates than those of other subtypes of soft tissue sarcoma (24\% vs. 10.4\%), but the differences were not statistically significant $(p=0.06)$. In addition, patients with uterine LMS had a longer survival than those with other subtypes of soft tissue sarcoma $(\mathrm{p}=0.01)$. In our study, subtypes of LMS affected approximately $26 \%$ of our patients and the response rate in these patients was $26.3 \%$. In our analysis of prognosticators of PFS, patients with LMS had significant differences, with a hazard ratio of 0.693 (95\% CI, 0.493 to $0.975 ; \mathrm{p}<0.05)$ compared to those with other subtypes.

Another study using gemcitabine and docetaxel combination therapy for soft tissue sarcoma was an open randomized trial that compared the administration of gemcitabine alone versus gemcitabine and docetaxel combination in 122 patients with various histopathological forms of unresectable soft tissue sarcoma, excluding Kaposi's sarcoma and gastrointestinal stromal tumors [12]. In that study, gemcitabine

Table 5. Toxicity (\% person)

\begin{tabular}{|lccccc|}
\hline Toxicity & G1 & G2 & G3 & G4 & $\begin{array}{c}\text { Unknown } \\
\text { grade }\end{array}$ \\
Hematological & & & & & \\
$\quad$ Neutropenia & $5(2.3)$ & $10(4.5)$ & $40(18.3)$ & $38(17.4)$ & - \\
\hline Anemia & $33(15.1)$ & $60(27.4)$ & $32(14.6)$ & $1(0.5)$ & - \\
\hline Thrombocytopenia & $22(10.2)$ & $16(7.3)$ & $20(9.1)$ & $5(2.3)$ & - \\
\hline Febrile neutropenia & $2(0.9)$ & - & $11(5.1)$ & $8(3.7)$ & \\
Nonhematological & & & & & \\
\hline Nausea/Vomiting & $23(10.5)$ & $13(5.9)$ & $3(1.4)$ & - & $21(9.6)$ \\
\hline Mucositis & $22(10.1)$ & $8(3.7)$ & $1(0.5)$ & - & $1(0.5)$ \\
\hline Peripheral neuropathy & $21(9.6)$ & $11(5.0)$ & $2(0.9)$ & - & $1(0.5)$ \\
Fatigue & $18(8.2)$ & $8(3.7)$ & $1(0.5)$ & $2(0.9)$ & $6(2.7)$ \\
\hline Diarrhea & $12(5.5)$ & $6(2.7)$ & $2(0.9)$ & - & $3(1.4)$ \\
\hline Myalgia & $19(8.7)$ & $6(2.7)$ & $1(0.5)$ & $1(0.5)$ & $4(1.8)$ \\
\hline Anorexia & $15(6.9)$ & $6(2.7)$ & - & - & $1(0.5)$ \\
\hline Skin rash & $8(3.7)$ & $4(1.8)$ & $2(0.9)$ & - & - \\
General weakness & $3(1.4)$ & $4(1.9)$ & $3(1.4)$ & - & $5(2.3)$ \\
\hline Abdominal pain & $5(2.3)$ & $6(2.7)$ & - & - & - \\
\hline Dyspnea & $3(1.4)$ & $5(2.3)$ & - & - & $1(0.5)$ \\
\hline Insomnia & $2(0.9)$ & $1(0.5)$ & - & - & $2(0.9)$ \\
\hline Fever & $11(5.1)$ & $2(0.9)$ & - & - & $1(0.5)$ \\
\hline Constipation & $7(3.2)$ & $2(0.9)$ & - & - & $1(0.5)$ \\
\hline Alopecia & $3(1.4)$ & $2(0.9)$ & $1(0.5)$ & - & - \\
\hline
\end{tabular}

Values are presented as number $(\%)$. 
and docetaxel combination therapy resulted in a higher response rate than gemcitabine alone $(16 \%$ vs. $8 \%)$, and showed prolongation of PFS (6.2 months vs. 3.0 months) and overall survival (17.9 months vs. 11.5 months).

Other studies were a Japanese study that retrospectively analyzed the medical records of 64 patients with progressed soft tissue sarcoma [13], a German study that examined 17 patients with epithelioid sarcoma from three institutions [14], an American study of 19 patients with recurrent, refractory pediatric soft tissue sarcoma from a single pediatric hospital [15], a German study of 34 patients with soft tissue sarcoma from a single institution [16], as well as Korean studies that include a phase II clinical trial with 24 patients with soft tissue sarcoma from four institutions [17] and a retrospective study of 22 patients with soft tissue sarcoma from a single Korean institution [18].

The current study investigated the efficacy and safety of combined gemcitabine and docetaxel in 218 patients, representing all cases of its use for soft tissue sarcoma in Korea. However, it should be noted that this study has some limitations. First, this study was based on patient case report forms collected retrospectively for evaluation of the use of anticancer drug off-label therapy; therefore, there were qualitative limitations of the data (such as missing adverse reactions and dosage changes). In addition, despite the multicenter studies, a central review of responses and adverse reactions could not be conducted owing to limited human and material resources, although hematologic toxicity was evaluated directly at the central lab using the clinical data of each hospital. Thus, it seems that the number of patients who reported nonhematological toxicities was somewhat lower than that reported in previous prospective studies. Moreover, because the data were collected retrospectively, it should be noted that there was some heterogeneity in patient characteristics of histologic subtypes. For example, in this study data, there were 11 cases of uterine LMS among a total of 57 cases of LMS. The response rate was $27.2 \%$, with three patients showing PR responses. Moreover a total of 46 cases were classified as nonuterine LMS because their primary sites were locations such as the extremities, kidneys and abdominal retroperitoneum. Twelve of these patients showed PR results, corresponding to a $26.15 \%$ response rate. However, in this study, for nine of the 11 patients (81.8\%) who were identified as having a uterine subtype, this was at least the third line of chemotherapy, whereas 30 patients $(65 \%)$ with non-uterine LMS received this therapy as their second line of treatment. Taxane therapy can be selected as an off-label therapy for uterine sarcoma in Korea. Therefore, gemcitabine plus docetaxel therapy may have been administered more often for heavily treated uterine subtype than non-uterine subtypes. Because of this selection bias, it was difficult to compare the response rates between subgroups of LMS in this study. There were also some differences in administration and dosage depending on the protocols of each hospital, and detailed protocols such as administration of granulocyte colony-stimulating factor in the event of adverse reactions differed slightly from hospital to hospital. Despite these numerous limitations, this study is a largescale national study of patients with soft tissue sarcoma, which is difficult to evaluate using prospective designs owing to its rare occurrence, and our results reflect the clinical efficacy of the gemcitabine and docetaxel regimen practiced in Korean hospitals. Thus, we believe that our study provides valuable clinical insights. In this study, in addition to the patient case reports, specific details submitted to the Korea HIRA for the examination of anticancer drug benefits from each hospital and follow-up evaluations of death dates were reviewed to improve the quality of data for response rate and survival periods. The results of this study will facilitate identification of the disease characteristics and selection of anticancer chemotherapy in clinical settings in Korea. In the future, more effective and individualized treatment approaches need to be explored for each histopathological subtype of soft tissue sarcoma.

The results of this nationwide retrospective analysis of 218 patients from 20 medical centers representing all cases of the use of gemcitabine and docetaxel combination therapy for treating patients with advanced soft tissue sarcoma in Korea showed that this combination treatment was effective and its toxicity was manageable. In conclusion, for patients with advanced soft tissue sarcoma, especially LMS, gemcitabine and docetaxel combination therapy could be an important therapeutic option.

\section{Conflicts of Interest}

Conflict of interest relevant to this article was not reported.

\section{Acknowledgments}

The funding for this study was supported by the Health Insurance Review \& Assessment, Wonju, Gangwon, Korea.

\section{Author Details}

${ }^{1}$ Department of Pharmaceutical Management, Health Insurance Review \& Assessment, Wonju, ${ }^{2}$ College of Pharmacy, Health, Social \& Clinical Pharmacy, Chung-Ang University, Seoul, ${ }^{3}$ Division of Hematology-Oncology, Department of Medicine, Samsung Medical Center, Sungkyunkwan University School of Medicine, Seoul, ${ }^{4}$ Department of Oncology, Asan Medical Center, University of Ulsan College of Medicine, Seoul, ${ }^{5}$ Division of Hematology and Medical Oncology, Department of Internal Medicine, Seoul National University Bundang Hospital, Seoul National University College of 
Medicine, Seongnam, ${ }^{6}$ Division of Oncology / Hematology, Department of Internal Medicine, Korea University College of Medicine, Seoul, ${ }^{7}$ Cancer Deliberation Committee, Health Insurance Review \&
Assessment Service, Wonju, ${ }^{8}$ Department of Biostatistics, Korea University College of Medicine, Seoul, Korea

\section{References}

1. DeVita VT Jr, Lawrence TS, Rosenberg SA. DeVita, Hellman, and Rosenberg's cancer: principles and practice of oncology. 10th ed. Philadelphia, PA: Lippincott Williams \& Wilkins; 2014.

2. Clark MA, Fisher C, Judson I, Thomas JM. Soft-tissue sarcomas in adults. N Engl J Med. 2005;353:701-11.

3. Santoro A, Tursz T, Mouridsen H, Verweij J, Steward W, Somers $\mathrm{R}$, et al. Doxorubicin versus CYVADIC versus doxorubicin plus ifosfamide in first-line treatment of advanced soft tissue sarcomas: a randomized study of the European Organization for Research and Treatment of Cancer Soft Tissue and Bone Sarcoma Group. J Clin Oncol. 1995;13:1537-45.

4. Elias A, Ryan L, Sulkes A, Collins J, Aisner J, Antman KH. Response to mesna, doxorubicin, ifosfamide, and dacarbazine in 108 patients with metastatic or unresectable sarcoma and no prior chemotherapy. J Clin Oncol. 1989;7:1208-16.

5. Antman KH, Ryan L, Elias A, Sherman D, Grier HE. Response to ifosfamide and mesna: 124 previously treated patients with metastatic or unresectable sarcoma. J Clin Oncol. 1989;7: 126-31.

6. National Comprehensive Cancer Network. NCCN clinical practice guidelines in oncology (NCCN Guidelines): soft tissue sarcoma. Version 2 [Internet]. Fort Washington, PA: National Comprehensive Cancer Network [cited 2017 Mar 2]. Available from: http:// www.nccn.org/professionals/physician_gls/pdf/ sarcoma.pdf.

7. ESMO/European Sarcoma Network Working Group. Soft tissue and visceral sarcomas: ESMO Clinical Practice Guidelines for diagnosis, treatment and follow-up. Ann Oncol. 2014;25 Suppl 3:iii102-12.

8. Sheng JY, Movva S. Systemic therapy for advanced soft tissue sarcoma. Surg Clin North Am. 2016;96:1141-56.

9. Ratan R, Patel SR. Chemotherapy for soft tissue sarcoma. Cancer. 2016;122:2952-60.

10. Colosia A, Khan S, Hackshaw MD, Oglesby A, Kaye JA, Skolnik JM. A systematic literature review of adverse events asso- ciated with systemic treatments used in advanced soft tissue sarcoma. Sarcoma. 2016;2016:3597609.

11. Bay JO, Ray-Coquard I, Fayette J, Leyvraz S, Cherix S, PipernoNeumann $S$, et al. Docetaxel and gemcitabine combination in 133 advanced soft-tissue sarcomas: a retrospective analysis. Int J Cancer. 2006;119:706-11.

12. Maki RG, Wathen JK, Patel SR, Priebat DA, Okuno SH, Samuels B, et al. Randomized phase II study of gemcitabine and docetaxel compared with gemcitabine alone in patients with metastatic soft tissue sarcomas: results of sarcoma alliance for research through collaboration study 002. J Clin Oncol. 2007;25:2755-63.

13. Kaya AO, Buyukberber S, Ozkan M, Alkis N, Sevinc A, Ozdemir NY, et al. Efficacy and toxicity of gemcitabine plus docetaxel combination as a second line therapy for patients with advanced stage soft tissue sarcoma. Asian Pac J Cancer Prev. 2012;13:463-7.

14. Pink D, Richter S, Gerdes S, Andreou D, Tunn PU, Busemann C, et al. Gemcitabine and docetaxel for epithelioid sarcoma: results from a retrospective, multi-institutional analysis. Oncology. 2014;87:95-103.

15. Rapkin L, Qayed M, Brill P, Martin M, Clark D, George BA, et al. Gemcitabine and docetaxel (GEMDOX) for the treatment of relapsed and refractory pediatric sarcomas. Pediatr Blood Cancer. 2012;59:854-8.

16. Schmitt T, Kosely F, Wuchter P, Schmier JW, Ho AD, Egerer G. Gemcitabine and docetaxel for metastatic soft tissue sarcoma: a single center experience. Onkologie. 2013;36:415-20.

17. Lee EM, Rha SY, Lee J, Park KH, Ahn JH. Phase II study of weekly docetaxel and fixed dose rate gemcitabine in patients with previously treated advanced soft tissue and bone sarcoma. Cancer Chemother Pharmacol. 2012;69:635-42.

18. Lee HY, Shin SJ, Kim HS, Hong SJ, Han JW, Lim ST, et al. Weekly gemcitabine and docetaxel in refractory soft tissue sarcoma: a retrospective analysis. Cancer Res Treat. 2012;44:43-9. 